\title{
A comparison of the efficacy of multiple ultraviolet light room decontamination devices in a radiology procedure room - CORRIGENDUM
}

In the original published article by Cadnum et $\mathrm{al}^{1}$, figure 3.B was incorrectly repeated instead of figure $4 . \mathrm{B}$. The correct version of Figure 4 appears on the following page. The authors apologize for this error. 


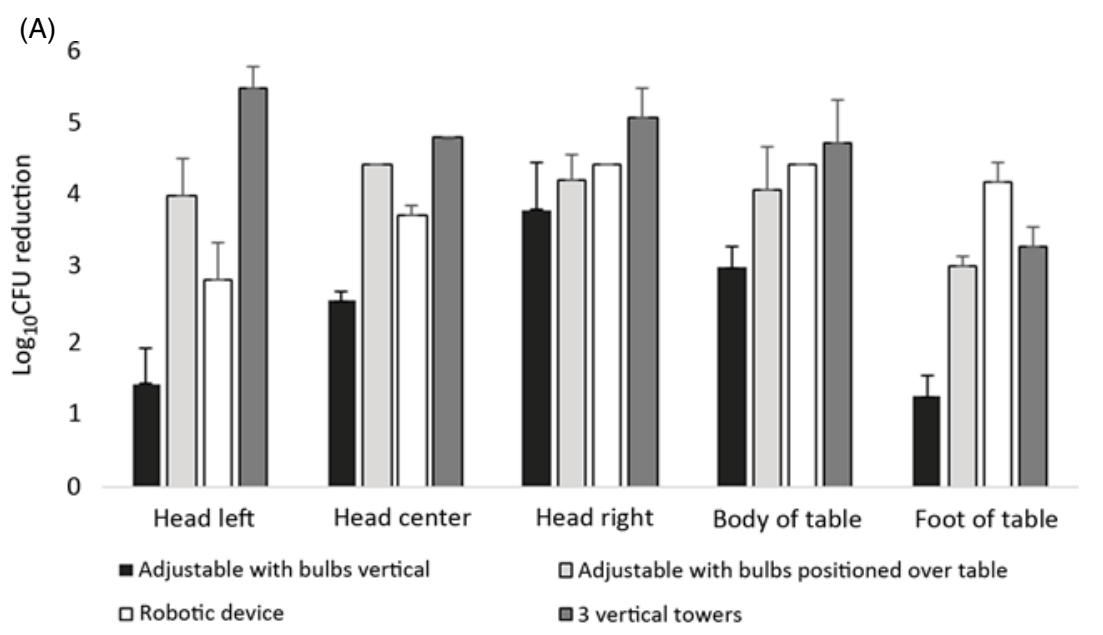

(B) 6

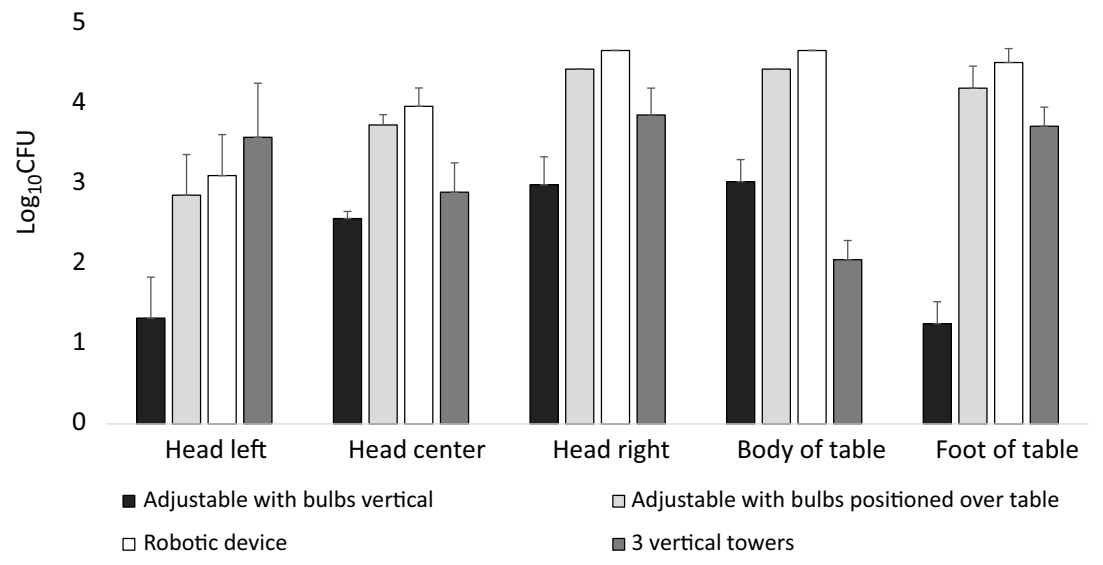

(C) 6

5

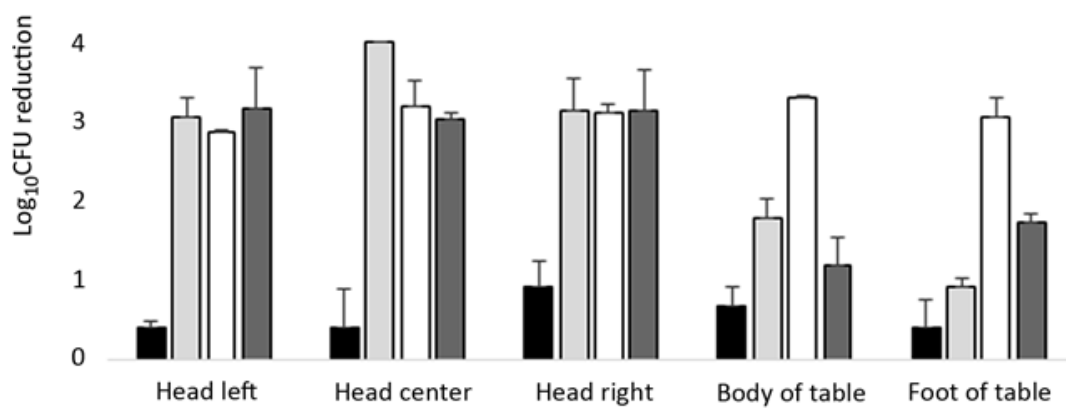

- Adjustable with bulbs vertical

口Adjustable with bulbs positioned over table

$\square$ Robotic device

$\square 3$ vertical towers

Fig. 4. Efficacy of 3 nonstandard ultraviolet light decontamination devices in reducing (A) methicillin-resistant Staphylococcus aureus (MRSA), (B) vancomycin-resistant Enterococcus, and (C) Clostridium difficile spores on 20-mm2 steel disk carriers placed on a radiology procedure table. The nonstandard devices included a device with 3 adjustable lamps that can be oriented to provide closer proximity to the surface of interest, a robotic device that moves along the side of the table during the treatment cycle, and a device that has 3 vertical towers that run simultaneously to reduce the impact of shadowing. The devices were operated for a 4-minute cycle and reductions in pathogens were measured in comparison to untreated controls. The means of data from triplicate experiments are presented. Error bars indicate standard deviation.

\section{Reference}

1. Cadnum, JL, Jencson, AL, Gestrich, SA, et al. A comparison of the efficacy of multiple UV light room decontamination devices in a radiology procedure room. ICHE 2019;40:158-163. 